\title{
Creating Websites: Adobe Go Live vs. LiveText
}

\author{
Donna F. Herring \\ Department of Educational Resources \\ College of Education and Professional Studies \\ Jacksonville State University \\ E-mail: dherring@jsu.edu \\ Charles E. Notar (Corresponding Author) \\ Department of Secondary Education \\ College of Education and Professional Studies \\ Jacksonville State University \\ Jacksonville, Alabama 36265-1602
}

Tel: 1-256-782-5832Ｅ-mail: cnotar@jsu.edu

\author{
Mrs. Judy McEntyre \\ Dalton State College \\ Educational Technology Training Center \\ Dalton, Georgia 30720 \\ E-mail: jmcentyre@dscettc.org
}

\begin{abstract}
This article reports student comments regarding their experiences in developing a website as part of a federally funded educational technology grant. The primary goal of the grant was to create a program where pre-service teachers developed competency in the use of technology for the academic success of their students. The students during the three year grant constructed websites for two years using Adobe Go Live and one year using LiveText. The two vehicles were compared on Ease of Use, Appearance, and Content with LiveText being preferred.
\end{abstract}

Keywords: Websites, LiveText, Adobe Go Live

\section{Creating Websites: Adobe Go Live vs. LiveText}

Objectives of a federally funded educational technology grant were the pre-service teachers will be able to:

(1) Create learning environments where advanced technology is used to teach content standards,

(2) Use a variety of classroom management techniques necessary for successful technology integration,

(3) Create technology connected lessons that include new designs for learning and enhanced pedagogy,

(4) Analyze student achievement/assessment data to make decisions for structuring technology connected lessons, and

(5) Create an educational website to enhance classroom learning.

Objective 5 was achieved through instruction the pre-service teachers received based on a state-developed educational technology program and the development of a website, integrating technology for the grade level of their clinical experiences. Pre-service teachers used the "Adobe-Go-Live" software program during the first two years of training, and LiveText during the third.

Evaluation of the grant activities included the following: 
- $\quad$ (LOTI) pretest/post-test process;

- university supervisor observation of the teachers' use of technology in classrooms during student teaching;

- $\quad$ a survey of pre-service teachers' students' computer abilities;

- $\quad$ intern pretest/post-test self evaluation of technological abilities;

- $\quad$ reflective journals;

- $\quad$ evaluator observation of pre-service teachers in their intern classroom;

- $\quad$ evaluator end-of-instruction interviews; and

- $\quad$ website evaluations.

Information for the article was gathered from pre-service teachers' reflective journals; evaluator observation of pre-service teachers in their intern classroom; evaluator end-of-instruction interviews; and website evaluations.

The information was compiled under three headings: Ease of Use, Appearance, and Content. Ease of use is defined as ability to use the software without assistance from the instructor.

Most students experienced problems when uploading or downloading GoLive files to the web. Many could not perform this activity on their own and needed assistance every time the site was uploaded or downloaded. The LiveText site was updated by simply saving. Students did not have a problem performing this task.

Adobe GoLive required an organized file structure for the site to work while LiveText did not. Students often deleted part of the structure or saved files into the wrong folder. By moving files or folders, it was easy to cause a broken link in GoLive. Again, this was not an issue in LiveText.

GoLive was driven by tools or palettes where LiveText was very similar to MicroSoft Word. LiveText tools were very familiar and easy to use and did not require a technical background. Online, 24-7, technical help was available via LiveText but was not for GoLive.

LiveText is a web-based software package and did not require access to a school server. GoLive did require access to a local web server. The ETTC hosted the GoLive websites but expected students to gain access to a server during their first year teaching. This did not happen in many cases.

Appearance is based on the appropriateness of the site for a particular grade level.

Students were able to add very creative backgrounds to GoLive. This was not an option in LiveText. However, the LiveText websites had a more consistent look that did the GoLive websites. Students spent more time working on content when using LiveText since they were limited in ability to use backgrounds and JAVA scripts with their site.

Content is based on the degree to which the site content supports and enhances the learning of the content standards.

The conclusion that can be drawn from the observations of the students is that LiveText provided the students with an easier to use, content-rich, standards-based website. Because of the ease of use, the LiveText websites were kept current and correlated well with the curriculum. The LiveText Rubric tool and the use of United Streaming Video within the software were not available as tools inside the GoLive software and added several dimensions to the students' lesson plans.

\section{References}

Bannister, S., Vannatta, R. A., \& Ross, C. (2006). Testing electronic portfolio systems in a teacher education: Finding the right fit. Action in Teacher Education, 27(4), 81-90.

Carlson, J. (2001). Easy web-site plans. Macworld, 18(2), 78,-80.

Carnevale, D. (2004). Files not found: Web-hosting company loses data of students and professors. Chronicle of Higher Education, 50(32), A34-A34.

Dysart, J. (2007). The beauty of web templates. American School Board Journal, 194(11), 50-51.

Fleishman, G. (2002). Go dynamic with GoLive. Macworld, 19(11), 86-89.

Goldsborough, R. (2003). S Sprucing up a website. Reading Today, 20(6), 16.

Gordon, R. S. (2003). Computer media. Library Journal, 128(6), 122.

Holzberg, C. S. (2005). No code? No problem! Technology \& Learning, 25(8), 18-21.

Myung-Ah, L. \& Hare, M. K. (2007). A web-based learning portfolio system. The Journal of Physical Education, Recreation \& Dance, 78(9), 45-48.

O'Reilly, D. (2002) Top-notch tools for web development. PC World, 20(8), 56. 
Go Live

Uploading Difficulties

Downloading

Difficulties

Structure Required

Broken Links

Palette Driven

More technical

knowledge

Must have server

access

No 24-7 support

Hugh learning curve

\section{LiveText}

Save and Make Public

Available with Internet access

Not Required

Less of an issue

Very similar to MS

Word

No technical

knowledge necessary

Hosted by Livetext

Provides 24-7

No learning curve

Figure 1. Ease of Use

\section{Go Live}

Backgrounds

Inconsistency of page

look

\section{LiveText}

Option not available

Very consistent look

from page to page

Figure 2. Appearance 


\section{Go Live}

None

None

None

Update in class with

help

Less content rich

No K-12 student use

\section{LiveText}

Standards Tool

Rubric Generator Tool

United Streaming Videos

Update on their own

More content rich

Easy for K-12 student

projects

Figure 3. Content 